\title{
Stemming Cancer: Functional Genomics of Cancer Stem Cells in Solid Tumors
}

\author{
C. R. A. Regenbrecht • H. Lehrach • J. Adjaye
}

Published online: 17 June 2008

(C) Humana Press 2008

\begin{abstract}
Cancer stem cells (CSCs) were discovered about 15 years ago in hematopoietic cancers. Subsequently, cancer stem cells were discovered in various solid tumors. Based on parallels with normal stem cells, a developmental process of cancer stem cells follows paths of organized, hierarchical structure of cells with different degrees of maturity. While some investigators have reported particular markers as identification of cancer stem cells, these markers require further research. In this review, we focus on the functional genomics of cancer stem cells. Functional genomics provides useful information on the signaling pathways which are consecutively activated or inactivated amongst those cells. This information is of particular importance for cancer research and clinical treatment in many respects. (1) Understanding of self-renewal mechanisms crucial to tumor growth. (2) Allow the identification of new, more specific marker for CSCs, and (3) pathways that are suitable as future targets for anti-cancer drugs. This is of particular importance, because today's chemotherapy targets the proliferating cancer cells sparing the relatively slow dividing cancer stem cells. The first step on this long road therefore is to analyze genome-wide expression-
\end{abstract}

C. R. A. Regenbrecht $\cdot$ J. Adjaye $(\bowtie)$

Department of Vertebrate Genomics, Max Planck Institute for Molecular Genetics (Molecular Embryology and Aging group), Ihnestrasse 73,

14195 Berlin, Germany

e-mail: adjaye@molgen.mpg.de

H. Lehrach

Department of Vertebrate Genomics,

Max Planck Institute for Molecular Genetics,

Ihnestrasse 73 ,

14195 Berlin, Germany profiles within the same type of cancer and then between different types of cancer, encircling those target genes and pathways, which are specific to these cells.

Keywords Cancer stem cell · Functional genomics · Genome-wide expression-profiling · Cell of origin . Tumor hierarchy · Self-renewal · Pathway-analysis

\section{Introduction}

Since the beginning of microscopic examination of tumors, many researchers have investigated the relationship between cancer cells and connective tissue. Rudolf Virchow was convinced that carcinomas derive from connective tissue, but soon was disproved by the studies of Cornil, Thiersch, and Waldeyer. Still, no clue was provided to explain the invasion of the epithelium. Julius Cohnheim and Hugo Ribbert hypothesized that invasion of epithelia was only possible in the case of a primarily altered connective tissue. Ultimately the stroma determines malignant growth. From 1902, Max Borst finally formulated the views on tumor-stroma-relationship which are still valid today. He also postulated interrelationships between tumor and stroma, which nowadays can be proven using molecular biological approaches.

The term 'stem cell' is used to describe cells capable of both self-renewal and differentiation to one or more functional lineages [1-3]. Moving down the stem cell hierarchy, from zygote to fully differentiated cell, the cell begins to lose pluripotency and becomes more specialized in morphology and function. Every stem cell, regardless of type (embryonic or adult), depends on its niche, or microenvironment. The niche comprises both extrinsic and intrinsic signals that govern cell fate. 


\section{The Cancer Stem Cell Hypothesis}

Cancer is characterized by mutations that cause uncontrolled cell proliferation and the formation of tumors. The majority of mutations leading to cancer activate cell-cycle checkpoints, which in normal cells prevent hyper-proliferation. The cancer stem cell (CSC) hypothesis arises in part from the observation that cancer cell populations are not homogenous. In 1971, Park et al. [4] were able to show that although tumors arise from a single cell, the cells that constitute the tumor are not identical to each other. This evidence of a heterogeneous population led others to investigate the cellular hierarchy of cancers. The cancer stem cell hypothesis of tumor development and progression states that cancer stem cells, like normal stem cells in other tissues, are a minority of the tumor cell population. Furthermore, serial transplantation experiments suggest that they are the only cells that can maintain tumor-growth indefinitely. The remaining cells, though actively proliferating and making up the majority of the cells in the tumor, are also differentiating and destined to die. The self-renewal properties of CSCs are thus the real driving force behind tumor-growth. It is uncertain whether these cells are actually stem cells, or if they were originally normal cells that have acquired stem cell-like properties. If indeed these cells do originate from stem cells, then it will be important to determine whether they are stem cells or progenitor cells.

The identification of markers that allow the prospective isolation of CSCs from whole tumor tissues will enable us to increase our meager understanding of several important biological properties of CSCs. (a) What is the cell of origin for a given tumor? (b) What are the signaling pathways that drive self-renewal and/or differentiation of CSCs? (c) Are there genes uniquely expressed in CSCs that will allow targeted therapies to be developed? (d) What are the mechanisms by which CSCs escape conventional therapies and can we defeat these mechanisms? Side populations of cells with stem cell properties have been identified in many cancers [5-7]. Addressing these questions should lead to the development of therapies that target the CSC population and eliminate the 'engine' that drives tumors to grow, invade and seed metastatic lesions. A side population was identified among thyroid cancer cell lines for the first time in 2007. Approximately $0.25 \%$ of cells in the anaplastic thyroid carcinoma cell line were shown to be sidepopulation cells. Other thyroid cancer cell lines harbor an even smaller percentage of side-population cells [8].

The challenges involved in identifying a CSC population from a solid tumor, and recent successes in this area are described in this review, as well as early studies that represent the first steps toward understanding the biological properties of cancer stem cells.
Identification of Markers for Isolating Putative Cancer Stem Cells

The ultimate proof of the existence of a sub-population of tumor initiating cells came from their ability to recapitulate the generation of a continuously growing tumor [9]. While this property has been widely accepted, the question regarding the origin of CSCs has yet to be answered. CSCs may have originated from normal stem cells, or more differentiated cells that have lost their full pluripotent competence. These cells then re-acquired self-renewal capacity through multiple mutagenic events, thus making them the driving force of tumor-growth. The most common way to identify CSCs in patient-derived tissues is labeling isolated cells with antibodies against various cell-surface markers already known in normal stem cells (Table 1). Cells bearing these cell-surface markers can be isolated by FACS [10] or magnetic bead columns [11]. These enriched cell populations are then tested for their ability to initiate tumorigenesis in immune deficient mice. These cells must then exhibit all properties of the original cells including a fully heterogeneous progeny. In contrast to hematopoietic tumors, the challenge of identifying and characterizing CSCs in solid tumors is significant. In most cases the normal tissue developmental hierarchy has not yet been identified or characterized. This makes the selection of candidate markers more difficult, and therefore does not provide the basis for comparison between normal and malignant cells, as there are no means to identify the cell of origin. In vivo models of human solid tumors can also be challenging to achieve, as in some cases orthotopic injection is not feasible or is technically challenging (e.g. lung, colon, and bladder cancers). Al-Hajj et al. [12] were the first to identify and prospectively isolate a minority subpopulation of cells from a human solid tumor (breast cancer) that contained all of the in vivo tumor-forming abilities, while the remaining bulk of cells from those

Table 1 List of known cancer stem cell markers in various tissues

\begin{tabular}{lll}
\hline Tumor type & CSC phenotype & Reference \\
\hline Breast & $\mathrm{CD} 44^{+} \mathrm{CD} 24^{-} /$low & Hope et al. [83] \\
CNS & $\mathrm{CD} 133^{+}$ & Al-Hajj et al. [12] \\
Multiple myeloma & $\mathrm{CD} 138^{-}$ & Singh et al. [14] \\
Melanoma & $\mathrm{CD} 20^{+}$ & Reynolds and Weiss [22] \\
Prostate & $\mathrm{CD} 44^{+} \alpha 2 \beta 1^{+}$CD133 & Fang et al. [25]; \\
& & Collins et al. [27] \\
HNSCC & $\mathrm{CD} 44^{+}$ & Matsui et al. [15] \\
Colon & $\mathrm{CD} 133^{+}$ & Dalerba et al. [18] \\
Colon & $\mathrm{CD} 44^{+}$EpCam $^{+}$ & Li et al. [17] \\
& $\mathrm{CD} 166^{+}$ & \\
Pancreatic & $\mathrm{CD} 44^{+}$EpCam $^{+} \mathrm{CD} 24^{+}$ & Prince et al. [16] \\
\hline
\end{tabular}


tumors had none, even when injected at many-fold higher cell doses. Similar findings were published for human brain tumors (GBMs and medulloblastomas). For normal neuronal stem cells it was already known that these cells express the marker CD133 [13], and it was demonstrated that as few as $100 \mathrm{CD} 133^{+}$cells from human brain tumors could initiate new tumors in the brains of immune deficient mice, whereas $100000 \mathrm{CD} 133^{-}$cells could not initiate tumors [14]. It has been shown that multiple myeloma contains a rare subset of cells, defined by their lack of expression of the plasma cell marker CD138, that are clonogenic in vitro and tumorigenic in vivo [15]. More recently, similar findings have been made for HNSCC [16], pancreatic cancer [17] and colon cancer [18-20]. The cell-surface phenotypes of CSC populations from these human solid tumors are described in Table 1. There have also been compelling studies in which putative CSCs have been identified based on their ability to form colonies in vitro. Sphere-forming ability as a measure of stem cells was first developed for central nervous system (CNS) cells, where it has been shown that a subset of cells isolated from human fetal brain, and subsequently from human CNS tumors, can form spheres when cultured under the appropriate conditions [13, 14, 21-24]. These spheres can self renew in vitro, and differentiate into all of the neuronal lineages, both in vitro and in vivo. Importantly, it was subsequently demonstrated for brain tumors that the neurosphere-forming cells could be prospectively isolated from fresh tissue using the cell-surface marker CD133. These $\mathrm{CD} 133^{+}$cells did indeed initiate brain tumors in vivo, without any in vitro manipulation, indicating that they do in fact represent CSCs [23]. Fang et al. [25] have demonstrated that upon culturing of metastatic melanoma cell suspensions under appropriate conditions, a subset of cells could be propagated as nonadherent spheres, which could then be induced to differentiate in vitro and to generate tumors in vivo. However, a marker has yet to be identified which would allow the prospective isolation of melanoma sphere-forming cells from fresh tumors, though the authors provided some evidence to suggest that CD20 can enrich for the multipotent fraction from established sphere cultures and melanoma cell lines. However, the ability to isolate and assay sub-populations of cells from tumors that behave as CSCs is essential before performing characterizations such as gene expression profiling. Such an approach would avoid artifacts introduced by culturing cells for extended periods of time. Another tumor for which CSCs have been potentially identified based on in vitro assays is prostate cancer [26]. Cells that are $\mathrm{CD} 44^{+} \alpha 2 \beta 1^{+} \mathrm{CD} 133^{+}$possess a significant capacity for self-renewal, differentiation, and invasiveness in vitro [27]. In this case, it also remains to be seen whether these cells can reproduce the original tumor in vivo.

\section{Understanding the Biological Properties of CSC}

Once CSCs have been identified, the real toil of characterizing their molecular and biological properties begins. The ultimate aim is identifying ways to specifically target and eradicate these cells in cancer patients. This is a new field in which we have barely begun to scratch the surface because of the only very recent ability to prospectively isolate CSCs from various tumors. Some of the approaches towards gaining a deeper understanding of CSC biology that have been reported are described below.

What is the Biological Significance of Cancer Stem Cells in Tumor Progression?

If cancer is maintained by CSCs that are characterized by low rates of division and proliferation, it is clear that therapies such as chemotherapy or radiation, which target actively cycling cells, will be ineffective $[28,29]$. Conventional treatments may cause the tumor to shrink temporarily, but the cells withstanding these regimens will inevitably lead to recurrence of the tumor. Recent studies have shown that $\mathrm{CD} 133^{+}$ cells within the glioma mediate resistance to radiation, at least in part due to elevated DNA damage response and more rapid repair of the damaged DNA [30]. The percentage of $\mathrm{CD}_{133^{+}}$cells increased following high dose radiation in established glioma cell lines, short-term cultures of biopsy material, and xenograft tumor-bearing mice, resulting in more aggressive tumors on serial transplantation. It is not clear why radiation should stimulate $\mathrm{CD} 133^{+}$cell division.

Very high radiation killed some CD133-expressing cells, reflecting perhaps a degree of heterogeneity. Others have reported that CSC-like cells from breast cancer cell lines are more radio-resistant compared to the rest of breast cancer cells [31]. These non-adherent sub-populations were grown as spheroids in mammosphere media and contained a larger fraction of cells with the $\mathrm{CD} 44^{+} / \mathrm{CD} 24^{- \text {low }}$ phenotype [12]. When these cultures were irradiated in vitro, the mammospheres were radio-resistant and there was a concomitant increase in the percentage of $\mathrm{CD} 44^{+} / \mathrm{CD} 24^{-/ \text {low }}$ cells. This would suggest that the relative radioresistance of this subset may lead to their expansion during the course of radiotherapy.

Other studies have shown that $\mathrm{CD} 133^{+}$cells isolated from GBM short-term cultures overexpressed drug resistance genes, such as breast cancer resistance protein-1 (BCRP1), DNA mismatch repair genes such as O-6-methylguanineDNA methyltransferase (MGMT), as well as showing higher levels of antiapoptotic gene expression [32]. These CD133 cells were also significantly more resistant to chemotherapeutic agents including temozolomide, carboplatin, paclitaxel (Taxol), and etoposide (VP16) compared with CD133negative cells [33]. In addition, it has been shown that CD $133^{+}$CSCs isolated from GBMs are resistant to ionizing 
radiation because they are more efficient at repairing damaged DNA than the bulk of the tumor cells [30]. Caveats to this study include the fact that radiation was applied to single cells separated from each other and their stromal components. It is not known how similar the response of these cells would be if they were in their natural setting in vivo. The radiation response of cells under normoxic conditions in culture used in this paper may not reflect the response of cells in the hypoxic environment of a tumor [34]. Similar studies in breast cancer cell lines in vitro have shown that $\mathrm{CD} 44^{+} / \mathrm{CD} 24^{-}$are more radio-resistant and that this correlated to differences in DNA damage responsiveness [31]. Recently, Blazek et al. [35] demonstrated that CD133 ${ }^{+}$ cells sorted from medulloblastoma cell lines are more radioresistant and this effect could be enriched by hypoxia. This is contrary to the clinical findings that medulloblastomas are much more radio-sensitive than GBMs. The fact that $\mathrm{CD}_{133^{+}}$has been proposed to be the cell of origin for both tumors illustrates the problems faced by the CSC hypothesis that focuses too much on the tumor cell and ignores the contribution of the micro-environment.

The differences in treatment response may be explained by age; that is, a micro-environment from younger patients (medulloblastoma) versus micro-environment from older patients (GBMs). Nevertheless, these shortfalls support the observation that CSCs are important mediators of resistance to treatment. The question that remains to be answered is whether radiation-resistance is a general property of CSCs in all tumors, and if their presence in human tumors is predictive of radio-sensitivity.

\section{Global Gene Expression Profiling}

One approach to analyse the genome-wide transcription levels is to compare the CSC profile to that of the nonCSCs within the tumor, or to the transcriptome of corresponding normal tissue. Such a study has been performed for breast cancer, in which the gene expression profile of the CSC was compared to that of normal breast epithelium. This analysis led to the identification of 186 genes which are differentially expressed in the CSC population. Upon comparison with previously reported gene signatures in breast cancer, there was a significant association between the CSC signature and both overall and metastasis-free survival [36]. The finding that detectable expression of a specific set of CSC-related genes upon analysis of whole tumor tissue correlates with prognosis suggests that more aggressive tumors may contain a relatively higher percentage of CSC, possibly caused by mutations that arrest cells in an immature state of differentiation. Indeed, differentiation status correlates with prognosis in many cancers. A similar approach was taken by Shipitsin et al. [37]. In this study breast tumor cells with a CSC phenotype were analyzed for their global gene expression profile compared to the non-tumorigenic cells from the same tumors. The profiles found indicated that cells of the CSC phenotype expressed stem/progenitor-associated genes whilst the non-CSC expressed differentiation-associated genes. They also found a correlation of the gene expression signature of CSCs with patient clinical outcome. Furthermore, they identified a signaling pathway (TGF- $\beta$ ) specifically active in the CSC population, and found that inhibition of this pathway in vitro led to differentiation. These observations once again demonstrate that purification of CSC allows the identification of biological properties of cells that are unique and that may otherwise not have been revealed. They also found that $\mathrm{CD} 24^{+}$cells in one patient sample had an additional genetic change not present in the $\mathrm{CD} 44^{+} / \mathrm{CD} 24^{-}$cells. The authors state that it has not been proven that the $\mathrm{CD} 24^{+}$cells are the progeny of the $\mathrm{CD} 44^{+} /$ CD24 $4^{-}$cells; however this is not the case. In the study by Al-Hajj et al. [12], it was shown that tumors grown from the $\mathrm{CD} 44^{+} / \mathrm{CD} 24^{-}$tumorigenic population give rise again to the original tumor heterogeneity, including the $\mathrm{CD} 24^{+}$ cells. Thus the finding of genetic changes present in the $\mathrm{CD} 24^{+}$cells but not in the $\mathrm{CD} 44^{+} \mathrm{CD} 24^{-}$population, but not vice versa, does not question the validity of the CSC hypothesis as suggested by Shipitsin et al. It is conceivable that additional genetic changes could take place in differentiating progenitors that are the progeny of the CSC, or that clonal evolution could occur within the CSC population itself as the tumor grows and the CSC population expands. Thus, although the authors state that their data calls the CSC hypothesis into question, in our opinion their data elegantly supports it.

\section{Comparison of CD133 Gene Expression Profiles}

Recently some studies were published, focusing on genomewide expression profiling in various cancers. In this review, we have compared the data-sets of 2 different studies that have provided genome-wide expression data from $\mathrm{CD} 133^{+}$ cells. This approach was of particular interest in regard of our own work, where we analyze $\mathrm{CD} 133^{+}$cells from human glioblastomas (data not published). Both studies presented about 100 candidate-genes, which were differentially expressed in the $\mathrm{CD} 133^{+}$-fraction $[38,39]$. As illustrated by the Venn-diagram in Fig. 1a, the overlap consists of just 10 genes, shown to be differentially regulated between the studies. Despite the relatively small datasets and the different ways of analyses in the original studies, the public available Search Tool for the Retrieval of Interacting Genes/Proteins (STRING) provides evidence that 9 out of the 10 candidate genes have been shown to form an interactive network directly interact with each other (Fig. 1b). Upon expansion of 
Fig. 1 Comparison of datasets of two independent genomewide expression analyses of CD133 ${ }^{+}$CSCs. a Venn diagram illustrating the common genes in $\mathrm{CD}_{133^{+}}$cells. $\mathbf{b}$ nine of 10 genes from this common dataset form a network, as retrieved from STRING search (http:// www.string.embl.de) c An expanded view of the interactions facilitated by these 10 genes reveals that the receptor thyrosin kinase KIT connects proliferative pathways (JAK/STAT) to morphogenetic pathways (HOX, GATA2, MEIS1)

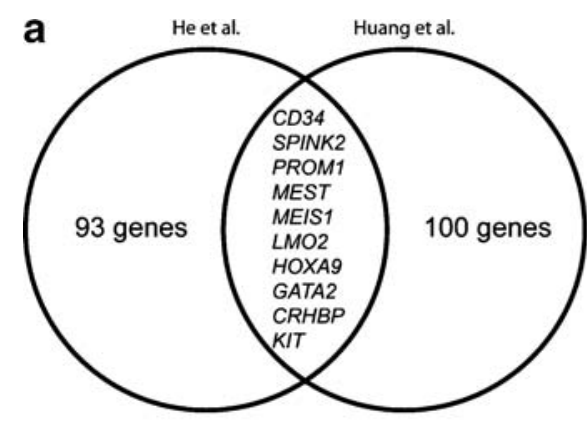

b

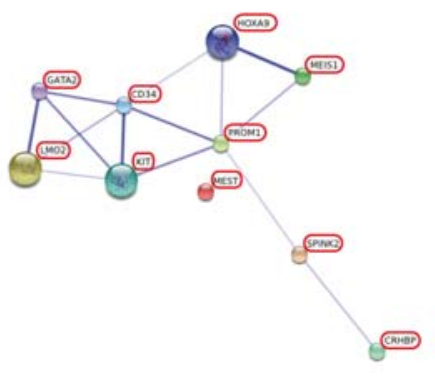

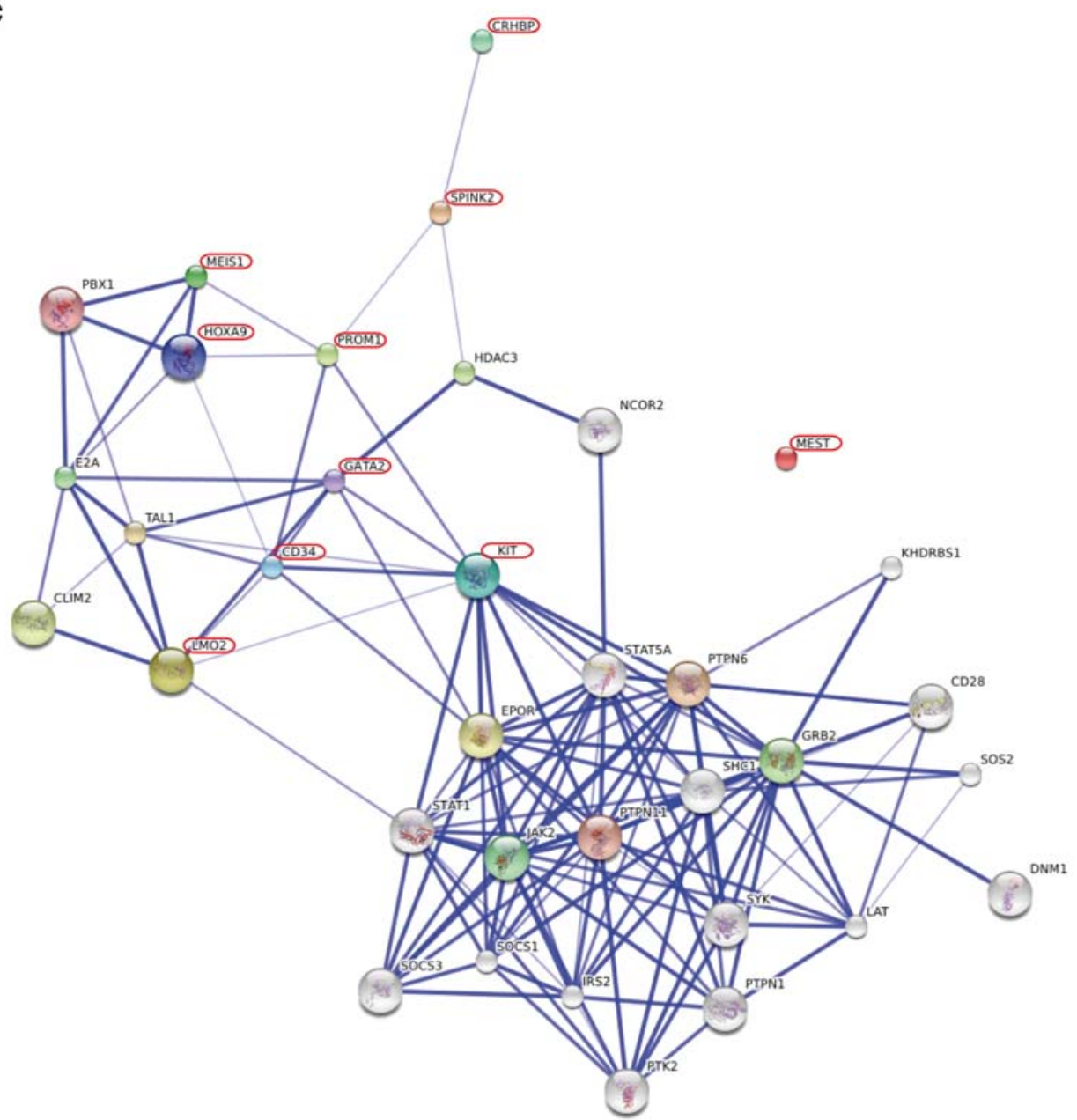

the network it becomes obvious that these target genes are positioned at the interface between proliferative pathways (JAK/STAT) and differentiating pathways (HOX, PBX, MEIS, GATA2) (Fig. 1c). Of importance in this cascade is the gene KIT. This gene encodes the receptor for stem cell factor (SCF) which is the human homolog of the protooncogene $C$-KIT. Mutations in KIT are associated with the outcome and progression in gliomas and acute myelogenous leukemia [40-42]. Mesoderm specific transcript homolog (MEST) does not connect to the proposed network of the other 9 genes. Despite that, in normal cells MEST is imprinted, and a loss of imprinting of MEST has been reported to associate with various cancers [43, 44]. Performing DAVID [45] annotation and KEGG [46] pathway analysis with the respective data-sets revealed significant enriched scores of numerous pathways. Amongst these are: Glycolisis/Gluconeogenesis (HSA00010), Cell Communication (HSA01430), Proteasome (HSA03050), Cell-Cycle (HSA04110), WNT-Signaling (HSA04310), TGF- $\beta$-Signaling (HSA04350) and Apoptosis (HSA04210). All of these pathways are known to play significant roles in various types of cancer development and progression. Proteins of all of these 
pathways are used to predict the outcome of disease. Finding these pathways in two independent genomic approaches overrepresented in the respective stem cell population makes it very tempting to speculate on the enormous significance of this small subpopulation of cells for the classification of the cancer. Besides the significance of these pathways in cancer, these pathways also play important roles in stem cells $[47,48]$. Comparative transcription profiling of normal and cancer stem cells is the way forward if we are to increase our meagre understanding of the molecular mechanisms underlying CSC proliferation.

\section{Identification of Cancer Relevant Pathways}

A microarray-based differential gene expression profile between side-population cells and non side-population cells has revealed several stem cell-associated genes $(A B C G 2$, $M Y C, J U N, F Z D 5, H E S 1$ and JAG1) as up-regulated in side-population cells. However, both side-population and non-sidepopulation cells derived from thyroid cancer cell lines can form tumors when injected into nude mice [8]. This finding suggests either today's markers are not a gold standard for isolating CSCs from bulk-tumor or that there is a delicate equilibrium between the tumor-cell populations maintained by autocrine and/or paracrine signaling. The cancer stem cell theory couples/reconciles the idea that stem cells are responsible for cancer with the hypothesis that distinct mutations in signaling pathways are involved in tumorgenicity. Signaling pathways associated with ES cell proliferation and differentiation are particularly important to the cancer stem cell theory $[47,48]$. For example, the $\mathrm{Wnt} / \beta$-catenin pathway is involved in the maintenance and self-renewal of hematopoietic stem cells and progenitor cells; overexpression of WNT protein is also seen in numerous human cancers [49]. Moreover, Sonic-hedgehog, which is involved in maintaining hematopoietic stem cells and progenitor cells as well as brain development, is associated with brain tumors [50].

Identification of Self-Renewal Signaling Pathways in CSCs

In addition to gene expression profiling, CSCs have been demonstrated to express known stem-cell-associated genes by other methods, including qRT-PCR and immune-staining. Head and neck squamous cell carcinoma (HNSCC) CSCs differentially express $B M I 1$ in the $\mathrm{CD}_{4} 4^{+}$population compared to the $\mathrm{CD} 44^{-}$population, and pancreatic CSCs differentially express SHH. Studies have yet to be conducted to determine whether these genes are functionally important for formation of these human tumor types. Their known roles in the self-renewal of normal tissue stem cells and other CSC populations suggest that they may also be important in these tumors. For example, in mice, BMI1 is essential for the self-renewal of hematopoietic and neural stem cells $[51,52]$ and has been shown to be required for the self-renewal of leukemic stem cells [53]. BMI1 is overexpressed in human AML compared to normal bone marrow. The Hedgehog pathway has long been implicated in many different kinds of cancers [54] and more recently has been related specifically to human CSCs from multiple myeloma [55], breast cancer [56] and gliomas [57, 58]. The WNT signaling pathway has also been implicated in both stem cell self-renewal and cancer [59-61], and has been shown specifically to be active in the CSC population of human CML blast crisis patients [62]. BMPs and their antagonists are known to play important roles in regulating homeostasis of various organs and tissues via the control of differentiation, proliferation and apoptosis [63, 64]. They have also been implicated in several cancers [63, 65]. Piccirillo et al. [66] recently demonstrated that BMPs, in particular $B M P 4$, depleted the brain tumor stem-cell population in vitro via a pro-differentiation effect, and inhibited tumorigenicity in vivo [66]. There are several other signaling pathways including the NOTCH, PTEN, and HOX pathways $[67,68]$ that have known roles in maintenance and/or control of normal and CSC compartments in mice, as well as being implicated in cancer. To date there is no conclusive evidence of a possible functional role specifically in human CSC populations. The discovery of signaling pathways that play a functional role in CSC self-renewal is extremely important from a therapeutic perspective, as some of these pathways have known chemical inhibitors (e.g. the Hh pathway can be inhibited by cyclopamine treatment), or function as inducers of differentiation (e.g. BMPs) [48, 69].

\section{Identification of a CSC Micro-Environment}

Normal stem cells are dependent upon their microenvironment, or 'niche' to maintain their ability of asymmetric division [70]. It is well known that a large component of solid tumors is made up of stromal cells and may consist of a variety of different cell types, including inflammatory cells, vascular endothelial cells and fibroblasts. In fact these stromal cells are essential for tumorgrowth, mediated either through direct cell contacts and/or secreted factors $[71,72]$. In an effort to understand the role of the micro-environment, Sneddon et al. [63] conducted a study where they compared the gene expression profiles of neoplastic stromal cells versus stromal cells from nontumor skin. This led to identification of GREM1 and FST, which are BMP antagonists, both genes are differentially expressed in the "tumor-stroma". Furthermore, BMP2 and BMP4 were expressed in SCC tumor cell nests, and GREMLIN1 was able to prevent inhibition of SCC growth by BMP4 in vitro. GREMLIN1 is expressed by stromal 
cells in diverse human carcinomas, but not in corresponding normal tissues. This study suggests that the expression of factors by the tumor-micro-environment that regulate self-renewal may be a general feature of human cancer, and identification of such factors may lead to the identification of potential therapeutic targets. Prince et al. [16] demonstrated that the CSC-containing population in well and moderately differentiated HNSCC is physically located adjacent to stroma, further indicating that there may be important interactions between the CSC and the stroma in carcinomas. Brain tumor stem cells also appear to interact with a niche. Mouse neural stem cells have been shown to lie within a vascular niche in which endothelial cells regulate stem cell self-renewal [73, 74]. Therefore Calabrese et al. [75] investigated whether the $\mathrm{CD}_{133}{ }^{+} / \mathrm{Nestin}^{+}$cells that contain the CSC fraction are associated with vasculature and found that indeed they are. Furthermore, endothelial cells supply secreted factors that maintain the brain tumor stem cells in vitro in a selfrenewing and undifferentiated state. Finally, increasing the number of endothelial cells or blood vessels in xenografts expanded the CSC population and accelerated its growth, while anti-angiogenic therapies depleted the CSCs from the xenografts and arrested tumor-growth. Thus the concept of the role of a CSC niche and the potential of inhibiting its interactions with CSC populations appears to be an approach with a great deal of therapeutic potential, both for disease treatment and for chemoprevention [76]. Furthermore, the identification of the CSC niche should allow the development of in vitro assay systems that mimic the in vivo environment, providing more rapid results than the currently cumbersome and time-consuming in vivo assays, as well as allowing manipulations that are currently not feasible with in vivo systems. Ultimately, an understanding of the CSC niche in addition to the CSCs themselves for each different type of cancer should lead to a better understanding of the signals that are important for CSC self-renewal and/or differentiation in those cancers.

\section{Are CSCs Selectively Resistant to Conventional Therapies?}

Normal stem cells are known to be relatively quiescent, resistant to drugs and toxins through the expression of drug efflux pumps, and have an active DNA-repair capacity and resistance to apoptosis [77]. If CSCs share many of the properties of normal stem cells, as is hypothesized, this means that conventional chemo and radiation therapies, which target rapidly cycling cells, will lead to reduction of the tumor through killing of the progeny of the CSCs, but the CSCs themselves will remain unaffected. Furthermore, the presence of drug efflux pumps and DNA-repair mechanisms will make them additionally resistant to these forms of therapy. Several studies have been done which support this hypothesis. In the hematopoietic system, both normal and leukemic stem cells are largely quiescent and express drug efflux molecules such as ABCG2 [78, 79], and AML stem cells are selectively resistant to both daunorubicin and Ara-C [80, 81]. More recently, it was shown that $\mathrm{CD}_{133^{+}}$brain tumor stem cells are selectively resistant to radiation, both in vitro and in vivo, and the mechanism for this resistance was shown to be through preferential activation of the DNA damage checkpoint response and an increase in DNA-repair capacity [30]. By understanding the mechanisms that enable CSCs to resist conventional therapies, it may be possible to find ways to manipulate these cells to become sensitive to these therapies.

\section{Clinical Implications of Cancer Stem Cells}

If proven to be true, the cancer stem cell theory could profoundly affect how cancer is treated. The identification of cancer stem cells provides a specific target for chemotherapy and drugs, and it may even dictate the aggressiveness of the treatment. Currently, many common chemotherapy and radiation protocols target all dividing cells, regardless of whether or not they are cancerous. However, if the disease is due to cancer stem cells, this could be the wrong approach, because cancer stem cells are relatively quiescent, they may survive treatment intended to kill dividing cells. In this case, the patient may appear to have made a full recovery only to relapse years later when the cancer stem cells are reactivated. If this scenario is true, it is possible that today's cancer therapies are merely

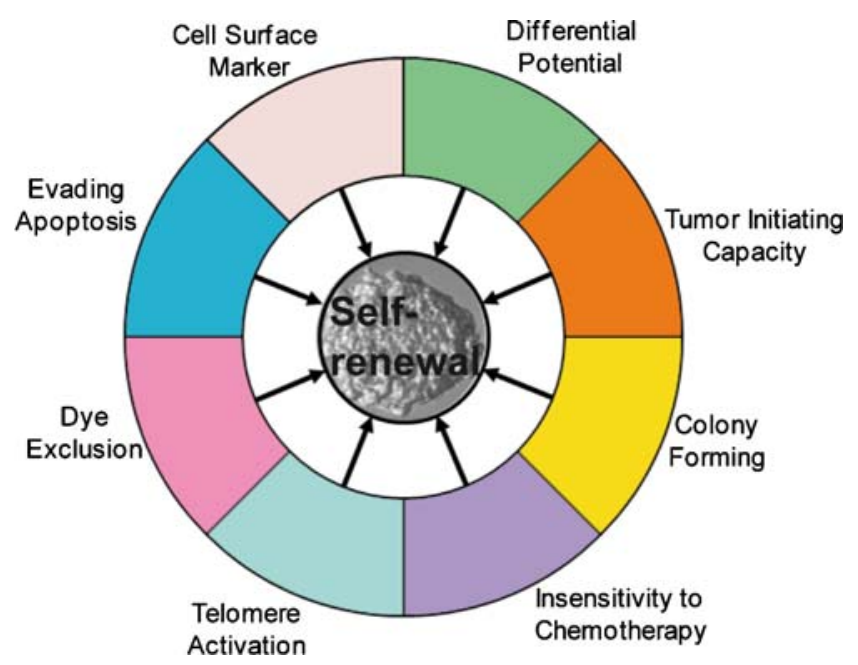

Fig. 2 Acquired capabilities of cancer stem cells. We suggest that most if not all cancer stem cells have acquired the same set of functional capabilities during their development, albeit through various mechanistic strategies. These properties provide potential leads for future cancer stem cell driven therapies 
helping patients 'maintain' their lives rather than getting to the root of the problem: cancer stem cells. Development of drugs that specifically target cancer stem cells may render cancer treatment more successful and efficient, and less toxic to the patient. For example, stem cells have active ATP-binding cassette transporters of specific types, which may increase the resistance of cancer stem cells to typical treatment options. Stem cell-specific targets such as these could be very useful in the design of new therapies. Although the cancer stem cell hypothesis is still being debated, the implications for future therapies are great, and it may even be able to change the way we diagnose and treat the disease.

\section{Conclusions}

The CSC field, particularly in the area of solid tumors, is still in its prime. On the basis of the highly recognized hallmark-paper by Hannahan \& Weinberg [82] we suggest a model highlighting the properties that are currently viewed as important for tumor growth, and that may represent therapeutic targets (Fig. 2). We are still in the very technically challenging stage of identifying the methods that will allow us to prospectively isolate CSCs from various solid tumors, and most importantly, to demonstrate in vivo that the populations we are looking at are truly CSCs. In the cases where CSCs have been identified, early studies have already shown that they possess important biological properties that directly relate to patient outcome. The breast CSC gene expression profile was shown to correlate with patient prognosis, and the brain CSC has been shown to be specifically resistant to radiation through DNA damage repair. In addition, specific signaling pathways have been shown to play a functional role in CSC self-renewal and/or differentiation, and early studies indicate that CSCs are associated with a micro-environmental niche. The mechanisms by which CSCs evade current therapies are being elucidated. These types of studies should lead to a better understanding of the molecular mechanisms controlling self-renewal and differentiation. The advent of new high-throughput technologies and the ability to apply them to small numbers of cells should lead to the identification of CSC-specific targets. The development of methods for the prospective isolation of CSCs is thus the first step, which then paves the way for a variety of approaches that could ultimately lead to CSC-specific therapies for cancer treatment.

The CSC hypothesis and emerging data on the functional properties of these cells have obvious therapeutic implications and raise the question whether we are targeting the right cells. Available data also suggest alternative strategies for homing on in CSCs in solid tumors, whereas animal studies suggest that CSCs can be induced to stop proliferating in a way that resembles differentiation of normal stem cells. Anti-cancer approaches aimed at the CSCs may not be suitable for all clinical settings. In GBM tumors, resistance to treatment is common to most cells within the cancer cell pool, so even proliferating cells survive the conventional treatments. In these cases, efficient control of the proliferating cells should be the primary objective, with targeting CSCs being a secondary aim. Although many uncertainties remain regarding the nature of CSCs in the CNS, accumulating data have demonstrated that there is a functional heterogeneity among the cancerous cells constituting a brain tumor. The focus of future research may therefore be to develop treatments that are modified according to this heterogeneity.

Acknowledgment The authors thank Prof. R Tikkanen and Dr. A Tirniceriu for critically reading the manuscript. This work was supported by the Max Planck Society and the Gisela Stadelmann-Stiftung.

\section{References}

1. Thomson, J. A., Itskovitz-Eldor, J., Shapiro, S. S., Waknitz, M. A., Swiergiel, J. J., Marshall, V., et al. (1998). Embryonic stem cell lines derived from human blastocysts. Science, 282, 1145-1147.

2. Gepstein, L. (2002). Derivation and potential applications of human embryonic stem cells. Circulation Research, 91, 866-876.

3. Zandstra, P. W., \& Nagy, A. (2001). Stem cell bioengineering. Annual Review of Biomedical Engineering, 3, 275-305.

4. Park, C. H., Bergsagel, D. E., \& McCulloch, E. A. (1971). Mouse myeloma tumor stem cells: a primary cell culture assay. Journal of the National Cancer Institute, 46, 411-422.

5. Chiba, T., Kita, K., Zheng, Y. W., Yokosuka, O., Saisho, H., Iwama, A., et al. (2006). Side population purified from hepatocellular carcinoma cells harbors cancer stem cell-like properties. Hepatology, 44, 240-251.

6. Kondo, T., Setoguchi, T., \& Taga, T. (2004). Persistence of a small subpopulation of cancer stem-like cells in the C6 glioma cell line. Proceedings of the National Academy of Sciences of the United States of America, 101, 781-786.

7. Patrawala, L., Calhoun, T., Schneider-Broussard, R., Zhou, J., Claypool, K., \& Tang, D. G. (2005). Side population is enriched in tumorigenic, stem-like cancer cells, whereas ABCG2+ and ABCG2- cancer cells are similarly tumorigenic. Cancer Research, $65,6207-6219$.

8. Mitsutake, N., Iwao, A., Nagai, K., Namba, H., Ohtsuru, A., Saenko, V., et al. (2007). Characterization of side population in thyroid cancer cell lines: cancer stem-like cells are enriched partly but not exclusively. Endocrinology, 148, 1797-1803.

9. Clarke, M. F., Dick, J. E., Dirks, P. B., Eaves, C. J., Jamieson, C. H., Jones, D. L., et al. (2006). Cancer stem cells-perspectives on current status and future directions: AACR Workshop on cancer stem cells. Cancer Research, 66, 9339-9344.

10. Woodward, W. A., Chen, M. S., Behbod, F., \& Rosen, J. M. (2005). On mammary stem cells. Journal of Cell Science, 118, 3585-3594.

11. Dou, J., Pan, M., Wen, P., Li, Y., Tang, Q., Chu, L., et al. (2007). Isolation and identification of cancer stem-like cells from murine 
melanoma cell lines. Cellular \& Molecular Immunology, 4, 467472.

12. Al-Hajj, M., Wicha, M. S., Benito-Hernandez, A., Morrison, S. J., \& Clarke, M. F. (2003). Prospective identification of tumorigenic breast cancer cells. Proceedings of the National Academy of Sciences of the United States of America, 100, 3983-3988.

13. Uchida, N., Buck, D. W., He, D., Reitsma, M. J., Masek, M., Phan, T. V., et al. (2000). Direct isolation of human central nervous system stem cells. Proceedings of the National Academy of Sciences of the United States of America, 97, 14720-14725.

14. Singh, S. K., Hawkins, C., Clarke, I. D., Squire, J. A., Bayani, J., Hide, T., et al. (2004). Identification of human brain tumour initiating cells. Nature, 432, 396-401.

15. Matsui, W., Huff, C. A., Wang, Q., Malehorn, M. T., Barber, J., Tanhehco, Y., et al. (2004). Characterization of clonogenic multiple myeloma cells. Blood, 103, 2332-2336.

16. Prince, M. E., Sivanandan, R., Kaczorowski, A., Wolf, G. T., Kaplan, M. J., Dalerba, P., et al. (2007). Identification of a subpopulation of cells with cancer stem cell properties in head and neck squamous cell carcinoma. Proceedings of the National Academy of Sciences of the United States of America, 104, 973-978.

17. Li, C., Heidt, D. G., Dalerba, P., Burant, C., Zhang, F. L., Adsay, V., et al. (2007). Identification of pancreatic cancer stem cells. Cancer Research, 67, 1030-1037.

18. Dalerba, P., Dylla, S. J., Park, I. K., Liu, R., Wang, X., Cho, R. W., et al. (2007). Phenotypic characterization of human colorectal cancer stem cells. Proceedings of the National Academy of Sciences of the United States of America, 104, 10158-10163.

19. O’Brien, C. A., Pollett, A., Gallinger, S., \& Dick, J. E. (2007). A human colon cancer cell capable of initiating tumour growth in immunodeficient mice. Nature, 445, 106-110.

20. Ricci-Vitiani, L., Lombardi, D. G., Pilozzi, E., Biffoni, M., Todaro, M., Peschle, C., et al. (2007). Identification and expansion of human colon-cancer-initiating cells. Nature, 445, $111-115$.

21. Hemmati, H. D., Nakano, I., Lazareff, J. A., Masterman-Smith, M., Geschwind, D. H., Bronner-Fraser, M., et al. (2003). Cancerous stem cells can arise from pediatric brain tumors. Proceedings of the National Academy of Sciences of the United States of America, 100, 15178-15183.

22. Reynolds, B. A., \& Weiss, S. (1996). Clonal and population analyses demonstrate that an EGF-responsive mammalian embryonic CNS precursor is a stem cell. Developments in Biologicals, 175, 1-13.

23. Singh, S. K., Clarke, I. D., Terasaki, M., Bonn, V. E., Hawkins, C., Squire, J., et al. (2003). Identification of a cancer stem cell in human brain tumors. Cancer Research, 63, 5821-5828.

24. Tamaki, S., Eckert, K., He, D., Sutton, R., Doshe, M., Jain, G., et al. (2002). Engraftment of sorted/expanded human central nervous system stem cells from fetal brain. Journal of Neuroscience Research, 69, 976-986.

25. Fang, D., Nguyen, T. K., Leishear, K., Finko, R., Kulp, A. N., Hotz, S., et al. (2005). A tumorigenic subpopulation with stem cell properties in melanomas. Cancer Research, 65, 9328-9337.

26. Mimeault, M., Hauke, R., Mehta, P. P., \& Batra, S. K. (2007). Recent advances in cancer stem/progenitor cell research: therapeutic implications for overcoming resistance to the most aggressive cancers. Journal of Cellular and Molecular Medicine, $11,981-1011$.

27. Collins, A. T., Berry, P. A., Hyde, C., Stower, M. J., \& Maitland, N. J. (2005). Prospective identification of tumorigenic prostate cancer stem cells. Cancer Research, 65, 10946-10951.

28. Berns, A. (2005). Stem cells for lung cancer? Cell, 121, 811-813.

29. Clarke, M. F. (2005). A self-renewal assay for cancer stem cells. Cancer Chemotherapy and Pharmacology, 56(Suppl 1), 64-68.

30. Bao, S., Wu, Q., McLendon, R. E., Hao, Y., Shi, Q., Hjelmeland, A. B., et al. (2006). Glioma stem cells promote radioresistance by preferential activation of the DNA damage response. Nature, 444 , 756-760.

31. Phillips, T. M., McBride, W. H., \& Pajonk, F. (2006). The response of $\mathrm{CD} 24(-/ \mathrm{low}) / \mathrm{CD} 44+$ breast cancer-initiating cells to radiation. Journal of the National Cancer Institute, 98, 1777-1785.

32. Liu, G., Black, K. L., \& Yu, J. S. (2006). Sensitization of malignant glioma to chemotherapy through dendritic cell vaccination. Expert Review of Vaccines, 5, 233-247.

33. Liu, G., Yuan, X., Zeng, Z., Tunici, P., Ng, H., Abdulkadir, I. R., et al. (2006). Analysis of gene expression and chemoresistance of CD133+ cancer stem cells in glioblastoma. Molecular Cancer, $5,67$.

34. Hambardzumyan, D., Squatrito, M., \& Holland, E. C. (2006). Radiation resistance and stem-like cells in brain tumors. Cancer Cell, 10, 454-456.

35. Blazek, E. R., Foutch, J. L., \& Maki, G. (2007). Daoy medulloblastoma cells that express CD133 are radioresistant relative to CD133- cells, and the CD133+ sector is enlarged by hypoxia. International Journal of Radiation Oncology, Biology, Physics, 67, 1-5.

36. Liu, R., Wang, X., Chen, G. Y., Dalerba, P., Gurney, A., Hoey, T., et al. (2007). The prognostic role of a gene signature from tumorigenic breast-cancer cells. New England Journal of Medicine, $356,217-226$.

37. Shipitsin, M., Campbell, L. L., Argani, P., Weremowicz, S., Bloushtain-Qimron, N., Yao, J., et al. (2007). Molecular definition of breast tumor heterogeneity. Cancer Cell, 11, 259-273.

38. He, X., Gonzalez, V., Tsang, A., Thompson, J., Tsang, T. C., \& Harris, D. T. (2005). Differential gene expression profiling of CD34+ CD133+ umbilical cord blood hematopoietic stem progenitor cells. Stem Cells and Development, 14, 188-198.

39. Huang, T. S., Hsieh, J. Y., Wu, Y. H., Jen, C. H., Tsuang, Y. H., Chiou, S. H. et al. (2008). Functional Network Reconstruction Reveals Somatic Stemness Genetic Maps and Dedifferentiation-Like Transcriptome Reprogramming Induced by GATA2. Stem Cells, 26 , 1186-1201.

40. Advani, A. S., Rodriguez, C., Jin, T., Jawde, R. A., Saber, W., Baz, R., et al. (2008). Increased C-kit intensity is a poor prognostic factor for progression-free and overall survival in patients with newly diagnosed AML. Leukemia Research, 32, 913-918.

41. Boissel, N., Leroy, H., Brethon, B., Philippe, N., de Botton, S., Auvrignon, A., et al. (2006). Incidence and prognostic impact of c-Kit, FLT3, and Ras gene mutations in core binding factor acute myeloid leukemia (CBF-AML). Leukemia, 20, 965-970.

42. Stanulla, M., Welte, K., Hadam, M. R., \& Pietsch, T. (1995). Coexpression of stem cell factor and its receptor c-Kit in human malignant glioma cell lines. Acta Neuropathologica, 89, 158-165.

43. Musci, T. J., Amaya, E., \& Kirschner, M. W. (1990). Regulation of the fibroblast growth factor receptor in early Xenopus embryos. Proceedings of the National Academy of Sciences of the United States of America, 87, 8365-8369.

44. Nishita, Y., Sado, T., Yoshida, I., \& Takagi, N. (1999). Effect of $\mathrm{CpG}$ methylation on expression of the mouse imprinted gene Mest. Gene, 226, 199-209.

45. Dennis Jr., G., Sherman, B. T., Hosack, D. A., Yang, J., Gao, W., Lane, H. C., et al. (2003). DAVID: Database for Annotation, Visualization, and Integrated Discovery. Genome Biology, 4, P3.

46. Wixon, J., \& Kell, D. (2000). The Kyoto encyclopedia of genes and genomes-KEGG. Yeast, 17, 48-55.

47. Babaie, Y., Herwig, R., Greber, B., Brink, T. C., Wruck, W., Groth, D., et al. (2007). Analysis of Oct4-dependent transcriptional networks regulating self-renewal and pluripotency in human embryonic stem cells. Stem Cells, 25, 500-510.

48. Greber, B., Lehrach, H., \& Adjaye, J. (2007). Silencing of core transcription factors in human EC cells highlights the importance 
of autocrine FGF signaling for self-renewal. BMC Developmental Biology, 7, 46.

49. Taipale, J., \& Beachy, P. A. (2001). The Hedgehog and Wnt signalling pathways in cancer. Nature, 411, 349-354.

50. Ruiz i Altaba, A., Sanchez, P., \& Dahmane, N. (2002). Gli and hedgehog in cancer: tumours, embryos and stem cells. Nature Reviews Cancer, 2, 361-372.

51. Molofsky, A. V., Pardal, R., Iwashita, T., Park, I. K., Clarke, M. F., \& Morrison, S. J. (2003). Bmi-1 dependence distinguishes neural stem cell self-renewal from progenitor proliferation. Nature, 425, 962-967.

52. Park, I. K., Qian, D., Kiel, M., Becker, M. W., Pihalja, M., Weissman, I. L., et al. (2003). Bmi-1 is required for maintenance of adult selfrenewing haematopoietic stem cells. Nature, 423, 302-305.

53. Lessard, J., \& Sauvageau, G. (2003). Bmi-1 determines the proliferative capacity of normal and leukaemic stem cells. Nature, 423, 255-260.

54. Kasper, M., Regl, G., Frischauf, A. M., \& Aberger, F. (2006). GLI transcription factors: mediators of oncogenic Hedgehog signalling. European Journal of Cancer, 42, 437-445.

55. Peacock, C. D., Wang, Q., Gesell, G. S., Corcoran-Schwartz, I. M., Jones, E., Kim, J., et al. (2007). Hedgehog signaling maintains a tumor stem cell compartment in multiple myeloma. Proceedings of the National Academy of Sciences of the United States of America, 104, 4048-4053.

56. Liu, S., Dontu, G., Mantle, I. D., Patel, S., Ahn, N. S., Jackson, K. W., et al. (2006). Hedgehog signaling and Bmi-1 regulate selfrenewal of normal and malignant human mammary stem cells. Cancer Research, 66, 6063-6071.

57. Bar, E. E., Chaudhry, A., Lin, A., Fan, X., Schreck, K., Matsui, W., et al. (2007). Cyclopamine-mediated hedgehog pathway inhibition depletes stem-like cancer cells in glioblastoma. Stem Cells, 25, 2524-2533.

58. Clement, V., Sanchez, P., de Tribolet, N., Radovanovic, I., \& Ruiz i Altaba, A. (2007). HEDGEHOG-GLI1 signaling regulates human glioma growth, cancer stem cell self-renewal, and tumorigenicity. Current Biology, 17, 165-172.

59. Reguart, N., He, B., Taron, M., You, L., Jablons, D. M., \& Rosell, R. (2005). The role of Wnt signaling in cancer and stem cells. Future Oncology, 1, 787-797.

60. Reya, T., Duncan, A. W., Ailles, L., Domen, J., Scherer, D. C., Willert, K., et al. (2003). A role for Wnt signalling in self-renewal of haematopoietic stem cells. Nature, 423, 409-414.

61. Reya, T., Morrison, S. J., Clarke, M. F., \& Weissman, I. L. (2001). Stem cells, cancer, and cancer stem cells. Nature, 414, 105-111.

62. Jamieson, C. H., Ailles, L. E., Dylla, S. J., Muijtjens, M., Jones, C., Zehnder, J. L., et al. (2004). Granulocyte-macrophage progenitors as candidate leukemic stem cells in blast-crisis CML. New England Journal of Medicine, 351, 657-667.

63. Sneddon, J. B., Zhen, H. H., Montgomery, K., van de Rijn, M., Tward, A. D., West, R., et al. (2006). Bone morphogenetic protein antagonist gremlin 1 is widely expressed by cancer-associated stromal cells and can promote tumor cell proliferation. Proceedings of the National Academy of Sciences of the United States of America, 103, 14842-14847.

64. Ye, L., Lewis-Russell, J. M., Kyanaston, H. G., \& Jiang, W. G. (2007). Bone morphogenetic proteins and their receptor signaling in prostate cancer. Histology and Histopathology, 22, 1129 1147.
65. Waite, K. A., \& Eng, C. (2003). From developmental disorder to heritable cancer: it's all in the BMP/TGF-beta family. Nature Reviews Genetics, 4, 763-773.

66. Piccirillo, S. G., Reynolds, B. A., Zanetti, N., Lamorte, G., Binda, E., Broggi, G., et al. (2006). Bone morphogenetic proteins inhibit the tumorigenic potential of human brain tumour-initiating cells. Nature, 444, 761-765.

67. Lobo, N. A., Shimono, Y., Qian, D., \& Clarke, M. F. (2007). The biology of cancer stem cells. Annual Review of Cell and Developmental Biology, 23, 675-699.

68. Rossi, D. J., \& Weissman, I. L. (2006). Pten, tumorigenesis, and stem cell self-renewal. Cell, 125, 229-231.

69. Greber, B., Lehrach, H., \& Adjaye, J. (2007). Fibroblast growth factor 2 modulates transforming growth factor beta signaling in mouse embryonic fibroblasts and human ESCs (hESCs) to support hESC self-renewal. Stem Cells, 25, 455-464.

70. Fuchs, E., Tumbar, T., \& Guasch, G. (2004). Socializing with the neighbors: stem cells and their niche. Cell, 116, 769-778.

71. Egeblad, M., Littlepage, L. E., \& Werb, Z. (2005). The fibroblastic coconspirator in cancer progression. Cold Spring Harbor Symposia on Quantitative Biology, 70, 383-388.

72. Kenny, P. A., Lee, G. Y., \& Bissell, M. J. (2007). Targeting the tumor microenvironment. Frontiers in Bioscience, 12, 3468-3474.

73. Palmer, T. D., Willhoite, A. R., \& Gage, F. H. (2000). Vascular niche for adult hippocampal neurogenesis. Journal of Comparative Neurology, 425, 479-494.

74. Shen, Q., Goderie, S. K., Jin, L., Karanth, N., Sun, Y., Abramova, N., et al. (2004). Endothelial cells stimulate self-renewal and expand neurogenesis of neural stem cells. Science, 304, 1338-1340.

75. Calabrese, C., Poppleton, H., Kocak, M., Hogg, T. L., Fuller, C., Hamner, B., et al. (2007). A perivascular niche for brain tumor stem cells. Cancer Cell, 11, 69-82.

76. Albini, A., \& Sporn, M. B. (2007). The tumour microenvironment as a target for chemoprevention. Nature Reviews Cancer, 7, 139-147.

77. Dean, M., Fojo, T., \& Bates, S. (2005). Tumour stem cells and drug resistance. Nature Reviews Cancer, 5, 275-284.

78. Steinbach, D., \& Legrand, O. (2007). ABC transporters and drug resistance in leukemia: was P-gp nothing but the first head of the Hydra? Leukemia, 21, 1172-1176.

79. Zhou, S., Schuetz, J. D., Bunting, K. D., Colapietro, A. M., Sampath, J., Morris, J. J., et al. (2001). The ABC transporter Bcrp1/ABCG2 is expressed in a wide variety of stem cells and is a molecular determinant of the side-population phenotype. Natural Medicines, 7, 1028-1034.

80. Costello, R. T., Mallet, F., Gaugler, B., Sainty, D., Arnoulet, C., Gastaut, J. A., et al. (2000). Human acute myeloid leukemia CD34+/ CD38- progenitor cells have decreased sensitivity to chemotherapy and Fas-induced apoptosis, reduced immunogenicity, and impaired dendritic cell transformation capacities. Cancer Research, 60, 44034411.

81. Guzman, M. L., Neering, S. J., Upchurch, D., Grimes, B., Howard, D. S., Rizzieri, D. A., et al. (2001). Nuclear factorkappaB is constitutively activated in primitive human acute myelogenous leukemia cells. Blood, 98, 2301-2307.

82. Hanahan, D., \& Weinberg, R. A. (2000). The hallmarks of cancer. Cell, 100, 57-70.

83. Hope, K. J., Jin, L. \& Dick, J. E. (2004). Acute myeloid leukemia originates from a hierarchy of leukemic stem cell classes that differ in self-renewal capacity. Nature Immunology, 5, 738-743. 